\title{
Minimal projections with respect to various norms
}

\author{
Asuman Güven AKSOY and Grzegorz LEWICKI
}

\begin{abstract}
We will show that a theorem of Rudin [37, 38, permits us to determine minimal projections not only with respect to the operator norm but with respect to quasinorms in operators ideals and numerical radius in many concrete cases.
\end{abstract}

\section{Introduction.}

Let $X$ be a Banach space over $\mathbb{R}$ or $\mathbb{C}$. We write $B_{X}$ for the closed unit ball and $S_{X}$ for the unit sphere of $X$. The dual space is denoted by $X^{*}$ and the Banach algebra of all continuous linear operators on $X$ is denoted by $B(X)$.

Definition 1.1. The numerical range of $T \in B(X)$ is defined by

$$
W(T)=\left\{x^{*}(T x): x \in S_{X}, x^{*} \in S_{X^{*}}, x^{*}(x)=1\right\} .
$$

The numerical radius of $T$ is then given by

$$
\|T\|_{w}=\sup \{|\lambda|: \lambda \in W(T)\} .
$$

Clearly, $\|\cdot\|_{w}$ is a semi-norm on $B(X)$ and $\|T\|_{w} \leq\|T\|$ for all $T \in B(X)$. The numerical index of $X$ is defined by

$$
n(X)=\inf \left\{\|T\|_{w}: T \in S_{B(X)}\right\}
$$

Equivalently, the numerical index $n(X)$ is the greatest constant $k \geq 0$ such that $k\|T\| \leq\|T\|_{w}$ for every $T \in B(X)$. Note also that $0 \leq n(X) \leq 1$, and $n(X)>0$ if and only if $\|\cdot\|_{w}$ and $\|\cdot\|$ are equivalent norms.

The concept of numerical index was first introduced by Lumer [31] in 1968. Since then much attention has been paid to this constant of equivalence between the numerical radius and the usual norm in the Banach algebra of all bounded linear

\footnotetext{
${ }^{0}$ Mathematics Subject Classification (2000): 41A35, 41A65, 47A12, 47H10.
}

Key words: Numerical radius, Minimal projection, Quasi-normed operator ideal . 
operators of a Banach space. Classical references here are [5], 6]. For recent results we refer the reader to [1],2] [16], 17], [19], [29], [32].

Let $\mathcal{L}$ denote the class of all operators between arbitrary Banach spaces. An Operator ideal $\mathcal{U}$ is, roughly speaking, a subclass of $\mathcal{L}$ such that

$$
\mathcal{U}+\mathcal{U}=\mathcal{U} \text { and } \mathcal{L} \circ \mathcal{U} \circ \mathcal{L}=\mathcal{U}
$$

The theory of normed operator ideals was founded by A. Grothendick and R. Schatten. Basic examples are the ideals of nuclear, compact, and absolutely summing operators. For more details on operator ideals see [36]. On every operator ideal there could be many different quasi-norms; however the "good" quasi-norms are selected by the completeness of the corresponding topology. Therefore, one can state that every operator ideal up to equivalence has one reasonable quasi-norm.

Definition 1.2. Let $\mathcal{U}$ be an operator ideal. A map

$$
\mathcal{Q}: \mathcal{U} \rightarrow \mathbb{R}^{+}
$$

is called a quasi-norm if the following conditions are satisfied:

1. $\mathcal{Q}\left(I_{V}\right)=1$, where $V$ denotes one dimensional Banach space.

2. There exists a constant $C \geq 1$ such that

$$
\mathcal{Q}\left(S_{1}+S_{2}\right) \leq C\left[\mathcal{Q}\left(S_{1}\right)+\mathcal{Q}\left(S_{2}\right)\right] \text { for } S_{1}, S_{2} \in \mathcal{U}(X, Y)
$$

3. If $T \in B\left(X_{0}, X\right), S \in B(X, Y)$ and $R \in B\left(Y, Y_{0}\right)$, then

$$
\mathcal{Q}(R S T) \leq\|R\| \mathcal{Q}(S)\|T\|
$$

Note that a quasi-operator ideal $(\mathcal{U}, \mathcal{Q})$ is an operator ideal $\mathcal{U}$ with quasi-norm $\mathcal{Q}$ such that $\mathcal{Q}(\lambda S)=|\lambda| \mathcal{Q}(S)$ for $S \in \mathcal{U}(X, Y)$ with $\lambda \in \mathbb{R}$. Furthermore, we have

$$
\|S\|_{w} \leq\|S\| \leq \mathcal{Q}(S)
$$

for all $S \in \mathcal{U}$.

If $X$ is a Banach space and $V$ is a linear, closed subspace of $X$, by $\mathcal{P}(X, V)$ we denote the set of all linear projections continuous with respect to the operator norm. Recall that an operator $P: X \rightarrow V$ is called a projection, if $\left.P\right|_{V}=i d_{V}$. A projection $P_{o} \in \mathcal{P}(X, V)$ is called minimal if

$$
\left\|P_{o}\right\|=\inf \{\|P\|: P \in \mathcal{P}(X, V)\}=\lambda(V, X) .
$$


Minimal projections were extensively studied by many authors in the context of functional analysis and approximation theory (see e.g. [1], 4], 7]-[10, 12][15, [18, [20]-30], 33]-35], 37, [39]-[41]). Mainly the problems of existence of minimal projections, uniqueness of minimal projections, finding concrete formulas for minimal projections and estimates of the constant $\lambda(V, X)$ were considered. It is worth noting that one of the main tools for finding minimal projection effectively is the so-called Rudin's Theorem (see [37] or [38]).

Now assume that $X$ is a Banach space, $N$ is any norm or semi-norm on an operator ideal $\mathcal{U}(X) \subset B(X)$ and $V$ is a subspace of $X$ such that $\left.i d\right|_{V} \in \mathcal{U}(X)$. Denote by $\mathcal{P}_{N}(X, V)$ the set of all linear projections from $X$ onto $V$ such that $P \in \mathcal{U}(X)$. Let us define

$$
\lambda_{N}(V, X)=\inf \left\{N(P): P \in \mathcal{P}_{N}(X, V)\right\} .
$$

We put $\lambda_{N}(V, X)=+\infty$ if $\mathcal{P}_{N}(X, V)=\emptyset$. A projection $P_{o} \in \mathcal{P}_{N}(X, V)$ is called $\mathrm{N}$-minimal if

$$
N\left(P_{o}\right)=\lambda_{N}(V, X) .
$$

In the following we consider $N$ as the numerical radius $\|\cdot\|_{w}$ or the quasi-norm norm $\mathcal{Q}($.$) and we will show that Rudin's Theorem can be applied to obtain N-$ minimal projections effectively. Although our proofs follow from Rudin's result without much difficulty, applications given in the last section justifiys the reason one may want to study minimal projections in this context.

It is worth mentioning that we do not know any paper (with the exception of [1]) concerning minimal projections with respect to norms different than the operator norm. In fact, in [1] a characterization of minimal numerical-radius extensions of operators from a normed linear space $X$ onto its finite dimensional subspaces and comparison with minimal operator-norm extension are given.

\section{Main Results}

One of the key theorems for minimal projections is due to W. Rudin (see page 127 of [37] or [38]). This theorem was motivated by an earlier result of Lozinkii (see e.g [11, p. 216 or [30]) concerning the minimality of the the classical n-th Fourier projection in $\mathcal{P}\left(C(2 \pi), \pi_{n}\right)$, where $C(2 \pi)$ denotes the space of all $2 \pi$-periodic realvalued functions equipped with the supremum norm and $\pi_{n}$ is the space of all trigonometric polynomials of degree less than or equal to $n$. The setting for this theorem is as follows. $X$ is a Banach space and $G$ is a compact topological group. Defined on $X$ is a set $\mathcal{A}$ of all bounded linear, bijective operators in a way that $\mathcal{A}$ is algebraically isomorphic to $G$. The image of $g \in G$ under this isomorphism will be denoted by $T_{g}$. We will assume that the map $G \times X \rightarrow X$ defined as $(g, x) \mapsto T_{g} x$ 
is continuous. A subspace $V$ of $X$ is called invariant under $G$ if $T_{g} V \subset V$ for all $g \in G$.

Theorem 2.1. Let $X$ and $G$ satisfy the above hypotheses, and let $V$ be a closed subspace of $X$ which is invariant under $G$. If there exists a bounded projection $P$ of $X$ onto $V$, then there exists a bounded projection $Q$ of $X$ onto $V$ which commutes with $G$.

The idea of the proof of the above theorem is to obtain $Q$ by averaging the operators $T_{g}^{-1} P T_{g}$ with respect to Haar measure $\mu$ on $G$. For details see [38.

Now assume $X$ has a norm which is an isometry for the maps in $\mathcal{A}$, and all the hypotheses of Theorem 2.1 are met, then we can claim the following corollary which is clearly a stronger result than Theorem 2.1 .

Corollary 2.2. If there is a unique projection $Q: X \rightarrow V$ which commutes with $G,\left(Q \circ T_{g}=T_{g} \circ Q\right)$ and $V$ is separable then for any $P \in \mathcal{P}(X, V)$ the projection $Q$ defined by

$$
Q x=\int_{G}\left(T_{g}^{-1} P T_{g}\right) x d \mu(g)
$$

is a minimal projection of $X$ onto $V$ with respect to the operator norm. Here $\mu$ denotes the normalized $(\mu(G)=1)$ Haar measure on $G$ and the above integral is understood as the Bochner integral. (By our assumptions concerning Rudin's Theorem, for any $x \in X$ the function $g \rightarrow\left(T_{g}^{-1} P T_{g}\right) x$ is continuous on $G$ and by the compactness of $G$ is Bochner integrable.)

For the proof of above corollary we refer to [38] and [14. For more details about Bochner integrals, see [14]. Now we show that Theorem 2.1] can be applied to find $N$-minimal projections.

Theorem 2.3. Suppose all the hypotheses of Theorem [2.1] are satisfied, $V$ is separable and the maps $\mathcal{A}$ are to be isometries. If $P$ is any projection in numerical radius from $X$ onto $V$, then the projection $Q_{P}$ defined as

$$
Q_{P} x=\int_{G}\left(T_{g}^{-1} P T_{g}\right) x d \mu(g)
$$

satisfies

$$
\left\|Q_{P}\right\|_{w} \leq\|P\|_{w}
$$

Here $\mu$ is the normalized Haar measure on $G$ and the above integral is understood as the Bochner integral. 
Proof. Consider $\left\|Q_{P}\right\|_{w}=\sup \left\{\left|x^{*}\left(Q_{P} x\right)\right|: x^{*}\left(Q_{P} x\right) \in W(Q)\right\}$, and since

$$
\left|x^{*}\left(Q_{P} x\right)\right|=\mid x^{*}\left(\int_{G}\left(T_{g}^{-1} P T_{g}\right) x d \mu(g) \mid\right.
$$

we have:

$$
\begin{gathered}
\left|x^{*}\left(Q_{P} x\right)\right|=\mid \int_{G}\left(x^{*} \circ\left(T_{g}^{-1}\right) P\left(T_{g} x\right) d \mu(g) \mid\right. \\
\leq \int_{G}\left|\left(x^{*} T_{g}^{-1}\right) P\left(T_{g} x\right)\right| d \mu(g) .
\end{gathered}
$$

But $\|x\|=1$ and $\left\|x^{*}\right\|=1$ implies that $\left\|T_{g} x\right\|=1$ and $\left\|x^{*} \circ T_{g^{-1}}\right\|=1$; moreover $\left(x^{*} \circ T_{g^{-1}}\right)\left(T_{g} x\right)=x^{*}(x)=1$, and thus

$$
\left|x^{*}(Q x)\right| \leq \int_{G}\|P\|_{w} d \mu(g) \leq\|P\|_{w}
$$

and $\|Q\|_{w} \leq\|P\|_{w}$ as required.

Theorem 2.4. Suppose that all the hypotheses of Theorem 2.3 are satisfied and that there exists exactly one projection $Q$ which commutes with $G$. Then $Q$ is a minimal projection with respect to the numerical radius.

Proof. Let $P \in \mathcal{P}(X, V)$. By the properties of the Haar measure, $Q_{p}$ given in Theorem 2.3 commutes with $G$. Since there is exactly one projection which commutes with $G, Q_{p}=Q$. By Theorem 2.3, $\|Q\|_{w} \leq\|P\|_{w}$, as required.

Now we consider the case of quasi-norms in operator ideals.

Theorem 2.5. Assume that $\mathcal{U}(X) \subset B(X)$ is an operator ideal and let $N$ be a quasi-norm on $\mathcal{U}(X)$. Suppose all the hypotheses of Theorem 2.1 are satisfied, $V$ is separable, $T_{g} \in \mathcal{U}(X)$ and $T_{g}$ is an isometry for any $g \in G$. For $P \in \mathcal{P}_{N}(X, V)$ define $Q_{P} \in \mathcal{P}_{N}(X, V)$ as

$$
Q_{P}=\int_{G} T_{g}^{-1} P T_{g} d \mu(g)
$$

where $\int_{G} T_{g}^{-1} P T_{g} d \mu(g)$ is understood as a linear operator from $X$ into $X$ defined by

$$
\left(\int_{G} T_{g}^{-1} P T_{g} d \mu(g)\right) x=\int_{G}\left(T_{g}^{-1} P T_{g}\right) x d \mu(g) .
$$

Assume additionally that

$$
N\left(\int_{G} T_{g}^{-1} P T_{g} d \mu(g)\right) \leq \int_{G} N\left(T_{g}^{-1} P T_{g}\right) d \mu(g) .
$$

Then $N\left(Q_{P}\right) \leq N(P)$. Here $\mu$ as before is the normalized Haar measure on $G$ and the above integral is understood as the Bochner integral. 
Proof. By the property (3) of Definition 1.2 for any $g \in G$ we have:

$$
N\left(T_{g}^{-1} P_{g} T_{g}\right) \leq\left\|T_{g}^{-1}\right\| N(P)\left\|T_{g}\right\|
$$

By our assumption

$$
\left.N\left(\int_{G} T_{g}^{-1} P T_{g} d \mu(g)\right) \leq \int_{G} N\left(T_{g}^{-1} P T_{g}\right) d \mu(g)\right) \leq N(P),
$$

as claimed.

Now we show that the assumption (2.1) is not very restrictive.

Remark 2.6. Let $\mathcal{U}(X) \subset \mathcal{B}(X)$ be an operator ideal. Assume that for any $L_{n}, L \in \mathcal{U}(X)$ if $L_{n} \rightarrow L$ pointwise $\left(\left\|L_{n} x-L x\right\| \rightarrow 0\right.$ for any $\left.x \in X\right)$, then

$$
N(L) \leq \limsup _{n} N\left(L_{n}\right) .
$$

In this case the assumption (2.1) is satisfied. It is not very difficult to check that (2.2) is satisfied for operator norms, p-summing norms, p-nuclear norms and pintegral norms (for precise definitions see e.g. (《2], Chapter III F).

Theorem 2.7. Suppose that all the hypotheses of Theorem 2.5 are satisfied and that there exists only one projection $Q$ which commutes with $G$. Then $Q$ is an $N$ minimal projection.

Proof. The proof follows the same lines as the proof of Theorem 2.4.

Now we show that the minimal projection obtained above by application of Theorem 2.1 is also a cominimal projection.

Theorem 2.8. (Cominimal projections) Assume that the assumptions of Theorem 2.4 are satisfied and let $I_{X}$ denoted the identity operator on $X$. Then

$$
N\left(I_{X}-Q\right) \leq N\left(I_{X}-P\right)
$$

for any $P \in \mathcal{P}_{N}(X, V)$, where $Q$ is defined in Theorem 2.4.

Proof. Let $Q_{P}$ be as in Theorem 2.3. Then $Q_{P}=Q$. Hence

$$
\begin{gathered}
N\left(I_{X}-Q\right)=N\left(I_{X}-\int_{G} T_{g}^{-1} P T_{g} d \mu(g)\right) \\
=N\left(I_{X} \int_{G} d \mu(g)-\int_{G} T_{g}^{-1} P T_{g} d \mu(g)\right)=N\left(\int_{G} T_{g}^{-1}\left(I_{X}-P\right) T_{g} d \mu(g)\right) .
\end{gathered}
$$


Now fix any $\left(x^{*}, x\right) \in S_{X^{*}} \times S_{X}$. Then

$$
\begin{gathered}
\left.\left|x^{*}\left(I_{X}-Q\right) x\right|=\mid \int_{G} x^{*} \circ\left(T_{g}^{-1}\left(I d_{X}-P\right) T_{g}\right) x\right) d \mu(g) \mid \\
\left.\leq \int_{G} \mid x^{*} \circ\left(T_{g}^{-1}\left(I d_{X}-P\right) T_{g}\right) x\right)\left|d \mu(g) \leq \int_{G}\right|\left(x^{*} \circ T_{g}^{-1}\right)\left(I_{X}-P\right)\left(T_{g} x\right) \mid d \mu(g) .
\end{gathered}
$$

Note that $\|x\|=1$ and $\left\|x^{*}\right\|=1$ implies that $\left\|T_{g} x\right\|=1$ and $\left\|x^{*} \circ T_{g^{-1}}\right\|=1$. Moreover $\left(x^{*} \circ T_{g^{-1}}\left(T_{g} x\right)=x^{*}(x)=1\right.$. Thus

$$
N\left(I_{X}-Q\right) \leq \int_{G} N\left(I d_{X}-P\right) d \mu(g) \leq N\left(I d_{X}-P\right)
$$

which completes the proof.

Combining the proofs of Theorem 2.5 and Theorem 2.8 one can easily get the following result.

Theorem 2.9. (Cominimal projections) Assume that the assumptions of Theorem 2.7 are satisfied and let $I_{X}$ denoted the identity operator on $X$. If $I d_{X} \in \mathcal{U}(X)$, then

$$
N\left(I_{X}-Q\right) \leq N\left(I_{X}-P\right)
$$

for any $P \in \mathcal{P}_{N}(X, V)$, where $Q$ is defined in Theorem 2.7.

Remark 2.10. In Theorem 2.8 and Theorem 2.9 we can replace $I d_{X}$ by any operator $S \in \mathcal{U}(X)$ satisfying

$$
S \circ T_{g}=T_{g} \circ S
$$

for any $g \in G$. Hence

$$
N(S-Q) \leq N(S-P)
$$

for any $P \in \mathcal{P}_{N}(X, V)$.

Remark 2.11. Assume that we have $W \subset S\left(X^{*}\right) \times S(X)$ with the following properties:

- For any $T: X \rightarrow X, T \in \mathcal{A}$ ( $T$ is an isometry)

- If $\left(x, x^{*}\right) \in W$ then $\left(x^{*} T^{-1}, T x\right) \in W$

Define on $\mathcal{L}(X)$ a semi-norm $\|\cdot\|_{W}$ given by

$$
\|L\|_{W}=\sup \left\{\left|x^{*} L x\right|:\left(x^{*}, x\right) \in W\right\}
$$

for any $L \in \mathcal{L}(X)$. Observe that for

$$
W=\left\{\left(x^{*}, x\right) \in S_{X^{*}} \times S_{X}: x^{*}(x)=1\right\},
$$

$\|L\|_{W}$ is equal to the numerical radius of $L$. Then the semi-norm $\|.\|_{W}$ satisfies Theorem 2.3, Theorem 2.4. Theorem 2.8 and Remark 2.10. 


\section{Applications}

We start with a classical example which explains the origins of the Rudin Theorem.

Example 3.1. Let $C(2 \pi)$ denote the set of all continuous, $2 \pi$-periodic functions and $\pi_{n}$ be the space of all trigonometric polynomials of order $\leq n(n \geq 1)$. A Fourier projection $F_{n}: C(2 \pi) \rightarrow \pi_{n}$ is defined by the formula:

$$
F_{n}(f)=\sum_{k=0}^{2 n}\left(\int_{0}^{2 \pi} f(t) g_{n}(t) d t\right) g_{k}
$$

where $\left(g_{k}\right)_{k=0}^{2 n}$ is an orthonormal basis in $\pi_{n}$ with respect to the scalar product

$$
<f, g>=\int_{[0,2 \pi]} f(t) g(t) d t .
$$

In [30] it is shown that $F_{n}$ is a minimal projection in $\mathcal{P}\left(C(2 \pi), \pi_{n}\right)$. The method of the proof is based on the Marcinkiewicz equality (see [11], p.233). For any $P \in \mathcal{P}\left(C(2 \pi), \pi_{n}\right), f \in C(2 \pi)$ and $t \in[0,2 \pi]$, let

$$
F_{n} f(t)=(1 / 2 \pi) \int_{[0,2 \pi]}\left(T_{g^{-1}} P T_{g} f\right) t d \mu(g) .
$$

Here $\mu$ is the Lebesgue measure and $\left(T_{g} f\right) t=f(t+g)$ for any $g \in \mathbb{R}$. Notice that $F_{n}$ is the only projection which commutes with $G$, where $G=[0,2 \pi)$ with addition $\bmod 2 \pi$. Consequently $F_{n}$ is an $N$-minimal projection as considered in Theorem 2.4 and Theorem 2.7.

Furthermore, it is known that (see[11],page 212) the operator norm of $F_{n}$ satisfies the following:

$$
\frac{4}{\pi^{2}} \ln (n) \leq\left\|F_{n}\right\| \leq \ln (n)+3 .
$$

In [1], it is shown that in cases of $L^{p}, p=1, \infty$, numerical radius extensions and minimal norm extensions are equal. Since $C(2 \pi) \subset L^{\infty}$, we also have

$$
\frac{4}{\pi^{2}} \ln (n) \leq\left\|F_{n}\right\|_{w} \leq \ln (n)+3
$$

It is worth noting that the Marcinkiewicz equality holds true if we replace $C(2 \pi)$ by $L^{p}[0,2 \pi]$ for $1 \leq p \leq \infty$ or by Orlicz space $L^{\phi}[0,2 \pi]$ equipped with the Luxemburg or the Orlicz norm provided that $\phi$ satisfies the suitable $\Delta_{2}$ condition. Hence, Theorem 2.4 and Theorem 2.7 can be applied to the numerical radius and quasinorms generated by $L_{p}$ norm or the Luxemburg or the Orlicz norm. 
Now we consider a more general situation.

Example 3.2. Let $m, n \in \mathbb{N}, n<m$. Assume

$$
V=\operatorname{span}\left[\sin \left(k_{i} \cdot\right), \cos \left(k_{i} \cdot\right), i=1, \ldots, n\right]
$$

and let

$$
X=\operatorname{span}\left[\sin \left(k_{i} \cdot\right), \cos \left(k_{i} \cdot\right), i=1, \ldots, m\right],
$$

where $k_{i} \in \mathbb{N}$ and $k_{1}<k_{2} \ldots<k_{m}$. Assume that $G$ is as in Example 3.1, It is easy to see that the only projection from $X$ onto $V$ which commutes with $G$ is given by

$$
Q\left(\sin \left(k_{i} \cdot\right)=0, Q\left(\cos \left(k_{i} \cdot\right)=0\right.\right.
$$

for $i>n$. Assume that $\|\cdot\|_{X}$ is any norm on $X$ such that the mapping

$$
T_{g}:\left(X,\|\cdot\|_{X}\right) \rightarrow T_{g}:\left(X,\|\cdot\|_{X}\right)
$$

is a linear isometry for any $g \in G$. Then $Q$ is a $N$-minimal projection as considered in Theorem 2.4 and Theorem 2.7. Typical examples of $\|\cdot\|_{X}$ are the $L_{p}$-norms, the Luxemburg and the Orlicz norms. Also it is possible to replace $X$ by $L_{p}[0,2 \pi]$ for $1 \leq p \leq \infty$ or by Orlicz spaces $L^{\phi}[0,2 \pi]$.

The same situation holds true in the complex case with

$$
\begin{gathered}
X=S \operatorname{pan}\left[e^{i k_{j} t}: i=1, \ldots, m\right], \\
V=\operatorname{Span}\left[e^{i k_{j} t}: i=1, \ldots, n\right]
\end{gathered}
$$

and

$$
G=\left\{e^{i t}: t \in[0,2 \pi]\right\} .
$$

Also we can apply Theorem 2.4 and Theorem 2.7 in multi-dimensional settings (see e.g [24]).

Example 3.3. Let $X=L^{p}[0,2 \pi]$ and let $V=H^{p}[0,2 \pi]$ be the Hardy space for $1<p<\infty$. By the M. Riesz Theorem (see [38], p.152), it follows that $\mathcal{P}\left(L^{p}[0,2 \pi], H^{p}[0,2 \pi]\right) \neq \emptyset$ and that the projection $Q$ given by

$$
Q\left(e^{i k_{j} \cdot}\right)=0
$$

for $j<0$ is the only projection which commutes with $G=\left\{e^{i t}: t \in[0,2 \pi]\right\}$. Hence $Q$ is an $N$-minimal projection as considered in Theorem 2.4 and Theorem 2.7. 
Example 3.4. Let $M(n, m)$ be the space of all (real or complex) matrices of $n$ rows and $m$ columns. Denote by $M(n, 1)(M(1, m)$ respectively) the space of matrices from $M(n, m)$ with constant rows (constant columns respectively). Let $S_{n}$ be the group of permutations of the set $\{1, \ldots, n\}$. Let $G=S_{n} \times S_{m}$. For any $g=\sigma \times \gamma \in G$ define a mapping $T_{g}: M(n, m) \rightarrow M(n, m)$ by

$$
T_{g}(A)(i, j)=A(\sigma(i), \gamma(j))
$$

for any $A \in M(n, m), i=1, \ldots, n$ and $j=1, \ldots, m$. Let

$$
W=M(n, 1)+M(1, m) .
$$

It is easy to see that $T_{g}(W) \subset W$ for any $g \in G$. Now assume that

$$
X=(M(n, m),\|\cdot\|)
$$

where $\|\cdot\|$ is any norm such that the mappings $T_{g}$ are isometries on $G$. Typical examples of such norms are $l_{p}$-norms and the Luxemburg and Orlicz norms. In ([14], Chapter 9) it has been shown that there is the unique projection $Q$ which commutes with $G$ that is given by a formula

$$
Q e_{r s}(i, j)= \begin{cases}\frac{n+m+1}{n m} & i=r, j=s \\ \frac{m-1}{n m} & i \neq r, j=s \\ \frac{n-1}{n m} & i=r, j \neq s \\ \frac{-1}{n m} & i \neq r, j \neq s\end{cases}
$$

where $e_{r s}(i, j)=\delta_{r i} \delta_{r j}$. Hence $Q$ is an $N$-minimal projection in any case considered in Theorem 2.4 and Theorem 2.7.

Example 3.5. Let for $x \in \mathbb{R},[x]$ denote the integer part of $x$. The well-known Rademacher functions $r_{o}, r_{1}, \ldots$ defined by $r_{j}(t)=(-1)^{\left[2^{j} t\right]}$ for $0 \leq t \leq 1$, play an important role in many areas of analysis. Let

$$
\operatorname{Rad}_{n}=\operatorname{span}\left[r_{o}, \ldots, r_{n}\right] .
$$

Applying Theorem 2.4 and Theorem 2.7 we will find a $N$-minimal projection from $X=\operatorname{Rad}_{m}$ onto $\operatorname{Rad}_{n}$ for any $m>n$ with respect to the $L_{p}$-norm for $(1 \leq p<\infty)$. To do this, we need to define so-called dyadic addition on the interval [0,1]. Let $Q$ denote the set of all dyadic rationals from the interval $[0,1)$, i.e.

$$
Q=\left\{x \in \mathbb{R}: x=\frac{p}{2^{n}}, p \in \mathbb{N}, 0 \leq p<2^{n}\right\} .
$$


Note that any $x \in[0,1]$ can be writtten in the form

$$
x=\sum_{k=0}^{\infty} x_{k} 2^{-(k+1)},
$$

where each $x_{k}=0$ or 1 . For each $x \in[0,1] \backslash Q$ there is only one expression of this form. When $x \in Q$ there are two expressions of this form, one which terminates in 0 's and the other one which terminates in 1's. By the dyadic expansion of $x \in Q$ we shall mean the one which terminates in 0's. Now we can define the dyadic addition of two numbers $x, y \in[0,1]$ by:

$$
x \oplus y=\sum_{k=0}^{\infty}\left|x_{k}-y_{k}\right| 2^{-(k+1)} .
$$

Notice that $G=([0,1], \oplus)$ is a group. Indeed, $x \oplus 0=x$ and $x \oplus x=0$. Let us define a metric $d$ on $G$ by

$$
d(x, y)=\max \left\{\sum_{k=0}^{\infty}\left|x_{k}-y_{k}\right| 2^{-(k+1)},|x-y|\right\},
$$

where $x=\sum_{k=0}^{\infty} x_{k} 2^{-(k+1)}$ and $y=\sum_{k=0}^{\infty} y_{k} 2^{-(k+1)}$ It is easy to see that $(G, d)$ is a compact, topological group. Moreover, the normalized Haar measure on $G$ is precisely the Lebesgue measure on $[0,1]$. Also it is easy to see that for any $n \in \mathbb{N}$ and $x \in[0,1]$

$$
r_{n}(x \oplus y)=r_{n}(x) r_{n}(y)
$$

provided $x \oplus y \notin Q$. Moreover, by the properties of Haar measures, for any $g \in[0,1]$ the operator $T_{g}: L_{p}[0,1] \rightarrow L_{p}[0,1]$ given by

$$
\left.\left(T_{g} f\right) x\right)=f(x \oplus g)
$$

is a linear, surjective isometry.

Now we will show that if $f_{n}, f \in L_{p}[0,1],\left\|f_{n}-f\right\|_{p} \rightarrow 0$ and $\left|g_{n}-g\right| \rightarrow 0$, then

$$
\left\|T_{g_{n}}\left(f_{n}\right)-T_{g}(f)\right\|_{p} \rightarrow 0 .
$$

To do this note that

$$
\left\|T_{g_{n}}\left(f_{n}\right)-T_{g}(f)\right\|_{p} \leq\left\|T_{g_{n}}\left(f_{n}\right)-T_{g_{n}}(f)\right\|_{p}+\left\|T_{g_{n}}(f)-T_{g}(f)\right\|_{p} .
$$

Observe that by changing variable from $x$ to $x \oplus g_{n}$ we get

$$
\left\|T_{g_{n}}\left(f_{n}\right)-T_{g}(f)\right\|_{p}^{p}=\int_{[0,1]}\left|f_{n}\left(x \oplus g_{n}\right)-f\left(x \oplus g_{n}\right)\right|^{p} d \mu(x)
$$




$$
=\int_{[0,1]}\left|f_{n}(x)-f(x)\right|^{p} d \mu(x)=\left\|f_{n}-f\right\|_{p}^{p} \rightarrow 0 .
$$

Notice that, if $f$ is a continuous function (and hence uniformly continuous on $[0,1])$, since $g_{n} \rightarrow g$, for any $\epsilon>0$ there exists $n_{o} \in \mathbb{N}$ such that for any $x \in[0,1]$ and $n \geq n_{o}\left|f\left(x \oplus g_{n}\right)-f(x \oplus g)\right| \leq \epsilon$. Consequently, $\left\|T_{g_{n}} f-T_{g} f\right\|_{p} \rightarrow 0$ for any $f \in C[0,1]$. By the Banach-Steinhaus Theorem, since $1 \leq p<\infty$, it follows that $\left\|T_{g_{n}} f-T_{g} f\right\|_{p} \rightarrow 0$, which shows (3.2).

Note that, since $\operatorname{Rad}_{n}$ is a finite-dimensional subspace, $\mathcal{P}\left(\operatorname{Rad}_{m}, \operatorname{Rad}_{n}\right) \neq \emptyset$. By (3.1) $T_{g}\left(\operatorname{Rad}_{n}\right) \subset \operatorname{Rad}_{n}$ for any $n \in \mathbb{N}$. Consequently, by Theorem 2.1, applying the fact that $g^{-1}=g$ for any $g \in G$, for any $P \in \mathcal{P}\left(X, \operatorname{Rad}_{n}\right)$ a projection

$$
Q_{p} f=\int_{[0,1]}\left(T_{g} P T_{g}\right) f d \mu(g) \in \mathcal{P}\left(X, \operatorname{Rad}_{n}\right)
$$

commutes with $G$. Now we show that there is exactly one projection from $X$ onto $\operatorname{Rad}_{n}$ which commutes with $G$. To do this, we show that for any $P \in \mathcal{P}\left(X, \operatorname{Rad}_{n}\right)$ $Q_{p}\left(r_{k}\right)=0$ for $m \geq k>n$. Accordingly we fix $x \in[0,1]$ and $g \in G$ with $x \oplus g \notin Q$. Note that

$$
\begin{gathered}
\left(T_{g} P T_{g} r_{k}\right) x=r_{k}(g)\left(T_{g} P T_{g} r_{k}\right) x=r_{k}(g)\left(T_{g}\left(\sum_{j=0}^{n} a_{j} r_{j}\right)\right) x \\
=r_{k}(g) \sum_{j=0}^{n} a_{j} r_{j}(x) r_{j}(g) .
\end{gathered}
$$

Observe that $\int_{[0,1]} r_{j}(g) r_{k}(g) d \mu(g)=0$ if $k \neq j$. Since for any $x \in[0,1]$

$$
\begin{gathered}
\mu(\{g \in G ; x \oplus g \in Q\})=0, \\
\left(Q_{p} r_{k}\right) x=\int_{[0,1]} r_{k}(g)\left(\sum_{j=0}^{n} a_{j} r_{j}(x) r_{j}(g)\right) d \mu(g)=0,
\end{gathered}
$$

which demonstrates our claim. Consequently, for any $P \in \mathcal{P}\left(\operatorname{Rad}_{m}, \operatorname{Rad}_{n}\right)$, and $f \in \operatorname{Rad}_{m}$,

$$
R_{n} f=Q_{p} f=\sum_{j=0}^{n}\left(\int_{[0,1]} r_{j}(t) f(t) d \mu(t)\right) r_{j}
$$

is an $N$-minimal projection as considered in Theorem 2.4 and Theorem 2.7, For more information about the nth Rademacher projection $R_{n}$ the reader is referred to [27]. 
Example 3.6. Let for $n \in \mathbb{N}, X_{n}=\mathcal{L}\left(\mathbb{R}^{n}\right)$. Set

$$
Y_{n}=\left\{L \in X^{n}: L=L^{T}\right\} .
$$

Let us equip $X_{n}$ with an operator norm determined by any symmetric norm $\|\cdot\|$ on $\mathbb{R}^{n}$. (We say that $\|\cdot\|$ is symmetric if

$$
\left\|\sum_{j=1}^{n} a_{j} e_{j}\right\|=\left\|\sum_{j=1}^{n} \epsilon_{j} a_{\sigma(j)} e_{j}\right\|
$$

for any $a_{1}, \ldots, a_{n} \in \mathbb{R}, \epsilon_{j} \in\{-1,1\}$ and any $\sigma$, where $\sigma$ is a permutation of $\{1, \ldots, n\})$.

Set for $L \in X_{n}$

$$
P(L)=\left(L+L^{T}\right) / 2 .
$$

It is clear that $P \in \mathcal{P}\left(X_{n}, Y_{n}\right)$. Moreover in [33] (see also [34]) it was shown applying Theorem 2.1 and Corollary 2.2 that $P$ is a minimal projection in $\mathcal{P}\left(X_{n}, Y_{n}\right)$. Hence $P$ is an $N$-minimal projection in any case considered in Theorem 2.4 and Theorem 2.7.

Example 3.7. Let $\left(s_{n}(S)\right)$ be a sequence associated with $S \in B(X, Y)$ satisfying certain conditions, one of which is

$$
s_{n}(R S T) \leq\|R\| s_{n}(S)\|T\|
$$

for $T \in B\left(X_{0}, X\right), S \in B(X, Y)$ and $T \in B\left(Y, Y_{0}\right)$. For a complete definition of $s$-numbers we refer to [36]. Furthermore, $s_{n}(S)$ is called an $n$th $s$-number of $S$ and various $s$-numbers generate different operator ideals. For example, for $0<p<\infty$, we call $S \in B(X, Y)$ a $\mathcal{C}_{p}^{s}$-operator if $\left(s_{n}(S)\right) \in \ell_{p}$. Setting

$$
\|S\|_{p}^{s}:=\left\{\sum_{1}^{\infty} s_{n}(S)^{p}\right\}^{1 / p}
$$

we obtain the quasi-normed operator ideal $\left(\mathcal{C}_{p}^{s},\|S\|_{p}^{s}\right)$. Thus one can apply Theorem 2.4 to the quasi norm $\|S\|_{p}^{s}$.

Note that in the context of Banach spaces there are several $s$-numbers, since there are certain rules assigning to every operator a decreasing sequence of numbers which characterize its approximation or compactness properties. The main examples of $s$-numbers are approximation numbers, Gelfand numbers, Kolmogorov numbers and Hilbert numbers. Thus, one can construct many operator ideals with quasi-norms depending on the particular $s$-number used. 


\section{References}

[1] A. G. Aksoy and B. L. Chalmers, Minimal numerical radius extension of operators, Proc. Amer. Math. Soc, Vol.135, no.4 (2007), 1039-1050 .

[2] A. G. Aksoy, E. Ed-dari, M. A. Khamsi, On the numerical index of vectorvalued function spaces, Linear Multilinear Algebra, 55, No.6, (2007), 507-513.

[3] M. Aleksiejczyk, On the diameter of the generalized numerical range, Demonstratio Math, 30 (1997), No. 1, 129-136.

[4] D. L. Berman, On the impossibility of constructing a linear polynomial operator furnishing an approximation of the order of best approximation, Dokl. Akad. Nauk SSSR, Vol. 120 (1958), 143-148.

[5] F. F. Bonsall and J. Duncan, Numerical ranges of Operators on Normed Spaces and of elements of Normed Algebras, London Math. Soc., Lecture Note Ser. 2, (Cambridge Univ. Press, 1971).

[6] F. F. Bonsall and J. Duncan, Numerical ranges II, London Math. Soc., Lecture Note Ser. 10 (Cambridge Univ. Press, 1971).

[7] B. L. Chalmers and G. Lewicki, Symmetric subspaces of $\ell_{1}$ with large projection constants, Studia Math. Vol.134, No. 2, (1999), 119-133.

[8] B. L. Chalmers and G. Lewicki, Symmetric spaces with maximal projection constant, J. Funct. Anal. Vol.200, (2003), 1-22.

[9] B. L. Chalmers and G. Lewicki, Three-dimensional subspace of $l_{\infty}^{(5)}$ with maximal projection constant, J. Funct. Anal., Vol. 2657, 553-592.

[10] B. L. Chalmers and F. T. Metcalf The determination of minimal projections and extensions in $L^{1}$, Trans. Amer. Math. Soc., Vol. 329, (1992), 289-305.

[11] E. W. Cheney Introduction to Approximation Theory Mc Graw-Hill, New York, 1966.

[12] E. W. Cheney and C. Franchetti, Minimal projections in $L_{1}$-space, Duke Math J. Vol. 43 (1976), 501-510.

[13] E. W. Cheney, C. R. Hobby, P. D. Morris, F. Schurer and D. E. Wulbert, On the minimal property of the Fourier projection, Trans. Amer. Math. Soc. Vol. 143 (1969), 249-258. 
[14] E. W. Cheney, W. A. Light, Approximation Theory in Tensor Product Spaces, Lecture Notes in Math., Vol 1169, Springer-Verlag, Berlin, 1985.

[15] E. W. Cheney and P. D. Morris, On the existence and characterization of minimal projections, J. Reine Angew. Math. Vol. 270, (1974), 61-76.

[16] E. Ed-dari, On the numerical index of Banach spaces, Linear Algebra Appl. 403 (2005), 86-96.

[17] C. Finet, M. Martín and R. Payá, Numerical index and renorming, Proc. Amer. Math. Soc. 131 (2003), no. 3, 871-877.

[18] S. D. Fisher, P. D. Morris and D. E. Wulbert Unique minimality of Fourier projections, Trans. Amer. Math. Soc. Vol. 265, (1981), 235-246

[19] K. E. Gustafson and D. K. M. Rao, Numerical range: The Field of Values of Linear Operators and Matrices, (Springer-Verlag UTX, New York, 1997).

[20] J. R. Isbell and Z. Semadeni, Projection constants and spaces of continuous functions, Trans. Amer. Math. Soc. Vol. 107 No. 1, (1963), 38-48.

[21] H. König Spaces with large projection constants, Israel J. Math., Vol. 50, (1985), $181-188$.

[22] H. König, C. Schuett, N. T. Jaegermann, Projection constants of symmetric spaces and variants of Khinchine's inequality, J. Reine Angew. math., Vol 511, (1999), 1-42.

[23] P. V. Lambert Minimum norm property of the Fourier projetion in spaces of continuous functions, Bull. Soc. Math. Belg. Vol. 21, (1969), 359-369.

[24] G. Lewicki, G. Marino, P. Pietramala, Fourier-type minimal extensions in real $L_{1}$-spaces, Rocky Mountain J. Math., Vol. 30, No. 3, (2000), 1025-1037.

[25] G. Lewicki Minimal extensions in tensor product spaces, J. Approx. Theory Vol. 97, (1999), 366-383.

[26] G. Lewicki, M. Prophet, Minimal multi-convex projections, Studia Math., Vol. 178, No.2, (2007), 71-91.

[27] G. Lewicki, L. Skrzypek, Chalmers-Metcalf operator and uniqueness of minimal projections, J. Approx. Theory, Vol. 148, (2007), 71-91.

[28] W. A. Light Minimal projections in tensor-product spaces, Math. Z. Vol. 191, (1986), No. 4, 633-643. 
[29] G. López, M. Martín and R. Payá, Real Banach spaces with numerical index 1, Bull. London Math. Soc. 31 (1999) 207-212.

[30] S. M. Lozinskii On a class of linear operators, Dokl. Acad. Nauk SSSR Vol. 61, No. 2 (1948), 193-196.

[31] G. Lumer, Semi-inner-product spaces, Trans. Amer. Math. Soc. 100 (1961), $29-43$.

[32] M. Martín, A survey on the numerical index of Banach space, Extracta Math 15 (2000), 265-276.

[33] D. Mielczarek, Minimal projections onto spaces of symmetric matrices, Univ. Iagel. Acta Math., Vol. 44, (2006), 69-82.

[34] D. Mielczarek, The unique minimality of an averaging projection, Monatsh. Math., Vol. 154, (2008), 157-171.

[35] W. Odyniec, G. Lewicki, Minimal Projections in Banach spaces, Lecture Notes in Mathematics, 1449, Springer-Verlag, 1990.

[36] A. Pietsch, Operator Ideals, North Holland, 1980.

[37] W. Rudin, Projections onto invariant subspaces, Proc. Amer. Math. Soc., 13, (1962), $429-432$.

[38] W. Rudin, Functional Analysis, McGraw Hill ( TMH Edition), 1974.

[39] L. Skrzypek, The uniqueness of minimal projections in smooth matrix spaces, J. Approx. Theory, Vol. 107, (2000), 315-336

[40] L. Skrzypek, Minimal projections in spaces of functions of $N$ variables, J. Approx Theory, Vol. 123, (2003), 214-231.

[41] B. Shekhtman, L. Skrzypek, Uniqueness of minimal projections onto twodimensional subspaces, Studia Math., Vol. 168, 92005), 273-284.

[42] P. Wojtaszczyk, Banach Spaces For Analysts, Cambridge Univ. Press, (1991).

Asuman Güven AKSOY

Claremont McKenna College

Department of Mathematics

Claremont, CA 91711, USA

E-mail: aaksoy@cmc.edu 
Grzegorz LEWICKI

Jagiellonian University

Department of Mathematics

Łojasiewicza 6, 30-348, Poland

E-mail: Grzegorz.Lewicki@im.uj.edu.pl 